\title{
Avaliação pela Tomografia de Coerência Óptica de Stent Nacional Recoberto com Polímero Biodegradável Eluidor de Sirolimus vs. Stent Eluidor de Biolimus A9 em Artérias Coronárias Porcinas
}

\author{
Celso Kiyochi Takimura', Micheli Zanotti Galon'1, Augusto Celso de Araújo Lopes Jr. ${ }^{1}$, Juliana Carvalho', \\ Suzane Kiss Ferreira', Márcio José Figueira Chaves1, Vera Demarchi Aiello1, Paulo Sampaio Gutierrez', \\ Francisco Rafael Martins Laurindoํ, Pedro Alves Lemos ${ }^{1}$
}

\section{RESUMO}

Introdução: Está em desenvolvimento em nosso meio o stent Inspiron ${ }^{\mathrm{TM}}$, um stent nacional metálico de cobalto-cromo recoberto com uma mistura de polímeros bioabsorvíveis exclusivamente em sua face abluminal e com liberação de sirolimus. O objetivo deste estudo foi comparar, em um modelo experimental, os achados da tomografia de coerência óptica dos stents Inspiron ${ }^{\mathrm{TM}}$ e BioMatrix ${ }^{\mathrm{TM}}$. Métodos: Os stents foram implantados em artérias coronárias porcinas. Cada indivíduo recebeu os dois tipos de stent, um em cada artéria (artérias descendente anterior e circunflexa), e após 28 dias foi realizado novo estudo angiográfico e empregada a tomografia de coerência óptica para avaliação da hiperplasia neointimal. Resultados: Sete porcos receberam 7 stents Inspiron $^{\mathrm{TM}}$ e 7 stents BioMatrix ${ }^{\mathrm{TM}}$. O diâmetro de referência dos vasos tratados foi de 2,16 \pm $0,37 \mathrm{~mm}$ e a relação balão de implante do stent/artéria foi de $1,17 \pm 0,16 \mathrm{~atm}$, sem diferença entre os grupos. A perda tardia intrastent foi de 0,53 $\pm 0,56 \mathrm{~mm}$ e de 0,32 \pm $0,37 \mathrm{~mm}(\mathrm{P}=0,43)$ para os stents Inspiron ${ }^{\mathrm{TM}}$ e BioMatrix ${ }^{\mathrm{TM}}$, respectivamente. Ocorreu um caso de reestenose angiográfica no grupo stent Inspiron ${ }^{\mathrm{TM}}(14 \%$ vs. $0 ; \mathrm{P}=0,2)$. A tomografia de coerência óptica evidenciou área neointimal intrastent no stent Inspiron ${ }^{T M}$ de 1,61 $\pm 0,57 \mathrm{~mm}^{2}$ e no stent BioMatrix $^{\mathrm{TM}}$ de $1,36 \pm 0,66 \mathrm{~mm}^{2}(P=0,47)$. A área neointimal porcentual foi de $31 \%$ no stent Inspiron ${ }^{\mathrm{TM}}$ vs. $23 \%$ no stent BioMatrix $^{\mathrm{TM}}(P=0,21)$. Conclusões: Após 28 dias de implante em artérias coronárias porcinas o stent Inspiron ${ }^{\mathrm{TM}}$ apresentou grau de hiperplasia neointimal intrastent semelhante ao do stent BioMatrix ${ }^{\top M}$.

DESCRITORES: Tomografia de coerência óptica. Stents farmacológicos. Neoíntima.

\section{ABSTRACT}

Assessment by Optical Coherence Tomography of a Brazilian Biodegradable Polymer-Coated Sirolimus Eluting Stent vs a Biolimus A9-Eluting Stent in Porcine Coronary Arteries

Background: Inspiron ${ }^{\mathrm{TM}}$, a chrome-cobalt sirolimus-eluting stent covered by a mixture of bioabsorbable polymers on its abluminal side is being developed in Brazil. Our objective was to compare in an experimental study the findings of optical coherence tomography for the Inspiron ${ }^{\mathrm{TM}}$ and BioMatrix $^{\top \mathrm{M}}$ stents. Methods: Stents were implanted in porcine coronary arteries. Each individual received two types of stents, one in each artery (left anterior descending and left circumflex artery). After 28 days, a new angiography was performed and optical coherence tomography was used to assess neointimal hyperplasia. Results: Seven Inspiron ${ }^{\top M}$ stents and 7 BioMatrix $^{\top M}$ stents were implanted in 7 domestic pigs. The reference diameter of the treated vessels was $2.16 \pm 0.37 \mathrm{~mm}$ and the balloon to artery ratio was $1.17 \pm 0.16$ atm, with no statistical difference between groups. In-stent late loss was $0.53 \pm$ $0.56 \mathrm{~mm}$ and $0.32 \pm 0.37 \mathrm{~mm}(\mathrm{P}=0.43)$ for the Inspiron $^{\mathrm{TM}}$ and BioMatrix ${ }^{\mathrm{TM}}$ stents, respectively. There was one case of angiographic restenosis in the Inspiron ${ }^{\mathrm{TM}}$ group (14\% vs 0 ; $\mathrm{P}=0.2$ ). Optical coherence tomography showed an instent neointimal area of $1.61 \pm 0.57 \mathrm{~mm}^{2}$ for the Inspiron ${ }^{\mathrm{TM}}$ stent and $1.36 \pm 0.66 \mathrm{~mm}^{2}$ for the BioMatrix ${ }^{\mathrm{TM}}$ stent $(P=0.47)$. The percentage of neointimal area was $31 \%$ for the Inspiron ${ }^{\mathrm{TM}}$ stent vs $23 \%$ for the Biomatrix ${ }^{\top M}$ stent $(P=0.21)$. Conclusions: Twenty-eight days after implantation in porcine coronary arteries the Inspiron ${ }^{\mathrm{TM}}$ stent showed a similar degree of neointimal hyperplasia as the Biomatrix ${ }^{\mathrm{TM}}$ stent.

KEY-WORDS: Tomography, optical coherence. Drug-eluting stents. Neointima.

\footnotetext{
1 Instituto do Coração do Hospital das Clínicas da Faculdade de Medicina da Universidade de São Paulo (InCor/HCFMUSP) - São Paulo, SP, Brasil. Correspondência: Celso Kiyochi Takimura - Rua Botelho, 155/162 - Vila Guarani - São Paulo, SP, Brasil - CEP 04313-200 E-mail: celsotakimura@hotmail.com

Recebido em: 8/3/2011 • Aceito em: 16/5/2011
} 
V ários tipos de stent farmacológico foram desenvolvidos desde a primeira avaliação dessas próteses em um estudo randomizado, publicado em 2002. ${ }^{1}$ Os primeiros stents farmacológicos disponibilizados para uso clínico - stents de primeira geração são constituídos de aço inoxidável e possuem polímero durável que liberam sirolimus ${ }^{2}$ (Cypher $^{\mathrm{TM}}$ - Cordis, Miami, Estados Unidos) ou paclitaxel ${ }^{3}$ (Taxus $^{\text {TM }}$ - Boston Scientific, Natick, Estados Unidos). Os stents farmacológicos que se seguiram - stents de segunda geração incorporaram uma série de inovações, como novas ligas metálicas, hastes e polímeros mais finos, polímeros posicionados apenas do lado abluminal das hastes, polímeros bioabsorvíveis, utilização de novas drogas antiproliferativas e stents com liberação de fármacos sem a necessidade de polímeros. Dois desses stents, constituídos de liga de cobalto-cromo, com hastes mais finas e flexíveis em relação aos stents de primeira geração, e com liberação de everolimus ${ }^{4}$ (Xience ${ }^{\mathrm{TM}}$ Abbott Laboratories, Abbot Park, Estados Unidos e Promus $^{\mathrm{TM}}$ - Boston Scientific) ou zotarolimus ${ }^{5}$ (Endeavor $^{\mathrm{TM}}$ Medtronic, Minneapolis, Estados Unidos) a partir de polímeros biocompatíveis e duráveis, foram aprovados pelo Food and Drug Administration para uso clínico.

Vários outros stents farmacológicos estão sob avaliação experimental e clínica e alguns deles estão liberados para uso clínico na Europa, na Ásia e no Brasil. Alguns desses stents são constituídos de novas ligas metálicas como platina-cromo ${ }^{6}$, não são recobertos por polímero $^{7,8}$ ou são bioabsorvíveis ${ }^{9}$ (estes últimos considerados stents de terceira geração). O stent BioMatrix ${ }^{\mathrm{TM}}$ (Biosensors International, Cingapura) é um stent de aço inoxidável inspirado no S-Stent ${ }^{\mathrm{TM}}$ (Biosensors International), recoberto com polímero biodegradável (PLA), exclusivamente em sua face abluminal, e que libera biolimus A9. ${ }^{10,11}$ Está em desenvolvimento em nosso meio o stent Inspiron ${ }^{\text {TM }}$ (Scitech Produtos Médicos Ltda., Goiânia, Brasil), um stent nacional metálico de cobaltocromo recoberto com uma mistura de polímeros bioabsorvíveis [poli(ácido láctico) e poli(ácido láctico-co-ácido glicólico)] também exclusivamente em sua face abluminal e com liberação de sirolimus. ${ }^{12-14}$

Este estudo teve como objetivo comparar, em um modelo experimental, os achados da tomografia de coerência óptica dos stents Inspiron ${ }^{\mathrm{TM}}$ e BioMatrix ${ }^{\mathrm{TM}}$, similares por serem revestidos em sua face abluminal por polímero biodegradável, diferenciando-se pela liga metálica e por liberarem sirolimus ou biolimus A9, respectivamente.

\section{MÉTODOS}

\section{Considerações éticas}

Em todos os procedimentos foram respeitadas as normas de proteção e cuidados para animais de experimentação estabelecidas nos "Princípios Éticos na Experimentação Animal" do Serviço de Apoio à Pesquisa e Experimentação Animal do Instituto do Coração da Faculdade de Medicina da Universidade de São Paulo, assim como no "Guide for the Care and Use of Laboratory Animals" (Institute of Laboratory Animal Resources, Comission on Life Sciences and National Research Council. National Academy Press, Washington, D.C., 1996) e nos "Princípios Éticos na Experimentação Animal" do Colégio Brasileiro de Experimentação Animal (COBEA). ${ }^{15}$

\section{Casuística}

Foram utilizados 7 porcos domésticos juvenis, nãoateroscleróticos, provenientes de granja comercial de reprodução.

\section{Medicações}

Um dia antes do procedimento foram administrados aos porcos, por via oral, ácido acetilsalicílico 600 mg e clopidogrel $150 \mathrm{mg}$. O protocolo de anestesia consistiu de medicação pré-anestésica (cetamina $3 \mathrm{mg} / \mathrm{kg}$ e midazolan $0,5 \mathrm{mg} / \mathrm{kg}$ por via intramuscular) seguida de infusão endovenosa de tiopental, entubação orotraqueal e início de ventilação mecânica com manutenção do nível anestésico com isofluorano.

\section{Técnica de implante dos stents e desenho do estudo}

Por dissecção da artéria femoral comum era introduzida, sob visão direta, uma bainha vascular $6 \mathrm{~F}$. Eram administradas 10.000 UI de heparina não-fracionada e, sob fluoroscopia, com o cateter-guia Judkins Right 46 F foi alcançada seletivamente a artéria coronária esquerda. A seguir, eram injetados pelo cateter $200 \mu \mathrm{g}$ de nitroglicerina, e nas artérias descendente anterior e circunflexa de cada indivíduo era implantado um stent (Inspiron $^{\mathrm{TM}}$ ou BioMatrix ${ }^{\mathrm{TM}}$ ), procurando uma relação balão de liberação do stent/artéria de 1,1/1. A escolha do tipo de stent implantado foi definida por sorteio prévio na etapa de planejamento do protocolo. Os tamanhos dos stents Inspiron ${ }^{\mathrm{TM}}$ disponíveis para implante foram de 2,5 mm e $3 \mathrm{~mm}$ de diâmetro por $16 \mathrm{~mm}$ de comprimento. Todos os stents BioMatrix ${ }^{\text {TM }}$ implantados tinham tamanho de $3 \times 14 \mathrm{~mm}$. Após o implante, os animais receberam ácido acetilsalicílico $100 \mathrm{mg}$ e clopidogrel $75 \mathrm{mg}$ por dia, e após 28 dias foram submetidos a reestudo angiográfico com avaliação pela tomografia de coerência óptica. As análises quantitativas de angiografia coronária e de tomografia de coerência óptica foram realizadas sem o conhecimento do tipo de stent em análise.

\section{Angiografia quantitativa coronária}

Foi utilizado o software Cardiovascular Angiographic Analysis System - CAAS II (Pie Medical Imaging, Maastrich, Holanda) para determinação do diâmetro de referência e do diâmetro luminal mínimo dos vasos-alvo, nas angiografias pré-implante do stent, pós-procedimento imediato e na angiografia de controle 
de 28 dias. Foi mensurado ainda o diâmetro do balão de liberação do stent em expansão máxima para cálculo da relação diâmetro do balão/diâmetro da artéria.

O ganho luminal agudo foi calculado pela diferença entre o diâmetro luminal mínimo pós-procedimento imediato e o diâmetro luminal mínimo pré-implante do stent. A perda luminal após 28 dias de implante dos stents foi calculada pela diferença entre o diâmetro luminal mínimo pós-procedimento imediato e o diâmetro luminal mínimo no reestudo. O porcentual de estenose foi calculado pelo resultado da equação: 1 - diâmetro luminal mínimo/diâmetro de referência medidas no reestudo. A reestenose angiográfica foi definida pela presença de estenose porcentual da lesão no reestudo angiográfico > 50\%.

\section{Tomografia de coerência óptica}

A tomografia de coerência óptica foi realizada utilizando-se o sistema OCT M2 (LightLab Imaging, Westford, Estados Unidos), com recuo automatizado do cateter de imagem ImageWire ${ }^{\mathrm{TM}}$ (LightLab Imaging) à velocidade de $1 \mathrm{~mm} / \mathrm{s}$ a $2 \mathrm{~mm} / \mathrm{s}$, após prévia oclusão proximal da artéria coronária com balão $\operatorname{Helios}^{\mathrm{TM}}$ (LightLab Imaging) e infusão de soro fisiológico. Utilizouse o software específico do equipamento de tomografia de coerência óptica para medida da área do lúmen e da área do stent em três imagens transversais de cada stent (terço proximal, terço médio e terço distal), com intervalo de análise e mensuração no stent Inspiron ${ }^{\mathrm{TM}}$ de $5,3 \mathrm{~mm}$ e de $4,6 \mathrm{~mm}$ no stent BioMatrix ${ }^{\mathrm{TM}}$. A área de neoíntima foi calculada pela diferença entre a área do stent e a área do lúmen. A área porcentual neointimal foi calculada a partir da relação entre a área neointimal e a área do stent. Malaposição de haste de stent foi definida pela visibilização de pelo menos uma haste de stent, com distância da parede do vaso maior que a espessura total do stent (espessura da haste somada à espessura da camada de polímero) informada pelo fabricante (stent Inspiron ${ }^{\mathrm{TM}} 80 \mu \mathrm{m}$ e stent BioMatrix ${ }^{\mathrm{TM}}$ $122 \mu \mathrm{m})$. Para avaliação de malaposição de hastes de stent foram analisadas todas as imagens transversais de cada stent e a intervalos de $1 \mathrm{~mm}$ foram contados o número de hastes visibilizadas relatando-se o número e o porcentual de hastes em malaposição.

Foi pesquisada ainda em cada sequência de imagens a presença de imagem sugestiva de trombo, definida como qualquer massa em protrusão para o lúmen com graus variáveis de atenuação da penetração da luz da tomografia de coerência óptica.

\section{Análise estatística}

Os dados são apresentados como média + desvio padrão (mediana) ou número (porcentagem). Foram utilizados para comparação das médias dos dois tipos de stent os testes de normalidade de Kolmogorov-Smirnov e de Shapiro-Wilk, seguidos pelo teste $t$ de Student para amostras independentes ou do teste de qui-qua- drado para comparação de variáveis categóricas. Valor de $\mathrm{P}<0,05$ foi considerado estatisticamente significante. As análises foram realizadas com a utilização do programa SPSS 11.0 (SPSS Inc., Chicago, Estados Unidos).

\section{RESULTADOS}

Todos os sete porcos sobreviveram ao procedimento de implante dos stents e foram submetidos a reestudo angiográfico e a estudo com tomografia de coerência óptica 28 dias após o implante.

A angiografia coronária quantitativa (Tabela 1) evidenciou artérias coronárias porcinas (artéria descendente anterior e artéria circunflexa) com diâmetro de referência de $2,16 \pm 0,37 \mathrm{~mm}$ e relação balão de implante do stent/artéria de 1,17 \pm 0,16 atm, sem diferença estatística entre os dois grupos. A perda tardia intrastent foi de $0,53+0,56 \mathrm{~mm}$ e de $0,32+0,37 \mathrm{~mm}$ $(P=0,43)$ e a porcentagem de estenose intrastent foi de $15 \pm 36 \%$ e $0 \pm 26 \%(P=0,36)$ para o stent Inspiron ${ }^{\mathrm{TM}}$ e para o stent BioMatrix ${ }^{\mathrm{TM}}$, respectivamente. Ocorreu um caso de reestenose angiográfica no grupo Inspiron ${ }^{\mathrm{TM}}$ (reestenose binária, 14\%) vs. nenhum caso de reestenose angiográfica no grupo BioMatrix ${ }^{\mathrm{TM}}(\mathrm{P}=0,2)$.

A tomografia de coerência óptica (Tabela 2) evidenciou área neointimal intrastent no stent Inspiron ${ }^{\mathrm{TM}}$ de 1,61 $\pm 0,57 \mathrm{~mm}^{2}$ e no stent BioMatrix ${ }^{\text {TM }}$, de 1,36 \pm $0,66 \mathrm{~mm}^{2}(\mathrm{P}=0,47)$. A área neointimal porcentual foi de $31 \%$ no stent Inspiron ${ }^{\mathrm{TM}}$ e de $23 \%$ no stent Biomatrix ${ }^{\mathrm{TM}}$ $(P=0,21)$.

De um total de 1.283 hastes visibilizadas em $105 \mathrm{~mm}$ de stents Inspiron ${ }^{\mathrm{TM}}$ e de 901 hastes em 96 mm de stents BioMatrix $^{\mathrm{TM}}$, foram observadas $4(0,31 \%)$ hastes em malaposição em um stent Inspiron ${ }^{\mathrm{TM}}$ e $2(0,22 \%)$ hastes em malaposição em 2 stents BioMatrix ${ }^{\mathrm{TM}}(P=0,69)$.

Nenhuma imagem sugestiva de trombo foi visibilizada nos stents dos dois grupos. As Figuras 1 e 2 mostram imagens de tomografia de coerência óptica representativas de um stent Inspiron ${ }^{\mathrm{TM}}$ e de um stent BioMatrix $^{\mathrm{TM}}$ após 28 dias de implante em artérias coronárias porcinas, respectivamente.

\section{DISCUSSÃO}

Os resultados deste estudo experimental apontam em primeiro lugar para a segurança conferida por esses dois tipos de stent farmacológico. Em segundo lugar observou-se semelhança entre os stents Inspiron ${ }^{\mathrm{TM}}$ e Biomatrix $^{\mathrm{TM}}$ no que se refere ao grau de hiperplasia neointimal após 28 dias de implante em artérias coronárias porcinas, avaliado pela tomografia de coerência óptica.

Essa semelhança do grau de hiperplasia neointimal segue-se à semelhança desses stents: recobertos com polímeros bioabsorvíveis e liberadores de fármacos da família limus apenas do lado abluminal. Por outro lado, apresentam diferenças em suas plataformas me- 
TABELA 1

Variáveis do procedimento e análise angiográfica quantitativa

\begin{tabular}{|c|c|c|c|c|}
\hline & $\begin{array}{c}\text { Total } \\
\text { (14 stents) }\end{array}$ & $\begin{array}{l}\text { Inspiron }{ }^{\mathrm{TM}} \\
\text { (7 stents) }\end{array}$ & $\begin{array}{l}\text { BioMatrix }^{\mathrm{TM}} \\
\text { (7 stents) }\end{array}$ & $\begin{array}{l}\text { Valor } \\
\text { de P }\end{array}$ \\
\hline \multicolumn{5}{|l|}{ Artérias, n } \\
\hline Artéria descendente anterior & 7 & 4 & 3 & 0,59 \\
\hline Artéria circunflexa & 7 & 3 & 4 & 0,59 \\
\hline Diâmetro dos stents, mm & $2,89 \pm 0,21(3)$ & $2,78 \pm 0,26(3)$ & $3 \pm 0(3)$ & 0,078 \\
\hline Comprimento dos stents, $\mathrm{mm}$ & $15 \pm 1(15)$ & $16 \pm 0(16)$ & $14 \pm 0(14)$ & $>0,99$ \\
\hline Diâmetro de referência, mm & $2,16 \pm 0,37(2,10)$ & $2,10 \pm 0,41(1,98)$ & $2,22 \pm 0,34(2,15)$ & 0,57 \\
\hline Pressão de liberação, atm & $7,8 \pm 3(8,5)$ & $7,4 \pm 3,3(9)$ & $8,2 \pm 2,8(8)$ & 0,62 \\
\hline Relação balão/artéria, atm & $1,17 \pm 0,16(1,12)$ & $1,19 \pm 0,16(1,15)$ & $1,15 \pm 0,17(1,11)$ & 0,68 \\
\hline DLM final, mm & $2,30 \pm 0,31(2,26)$ & $2,22 \pm 0,31(2,27)$ & $2,37 \pm 0,31(2,25)$ & 0,39 \\
\hline Ganho agudo, mm & $0,13 \pm 0,21(0,15)$ & $0,12 \pm 0,19(0,13)$ & $0,15 \pm 0,24(0,17)$ & 0,78 \\
\hline DLM tardio, mm & $1,86 \pm 0,62(2)$ & $1,68 \pm 0,72(2,02)$ & $2,04 \pm 0,49(1,99)$ & 0,3 \\
\hline Perda luminal intrastent, mm & $0,43 \pm 0,47(0,33)$ & $0,53 \pm 0,56(0,52)$ & $0,32 \pm 0,37(0,26)$ & 0,43 \\
\hline Perda luminal no segmento, mm & $0,59 \pm 0,50(0,56)$ & $0,57 \pm 0,58(0,52)$ & $0,61 \pm 0,46(0,73)$ & 0,88 \\
\hline Porcentagem de estenose intrastent, \% & $7 \pm 31(3)$ & $15 \pm 36(6)$ & $0 \pm 26(0)$ & 0,36 \\
\hline Porcentagem de estenose no segmento, \% & $15 \pm 27(9)$ & $17 \pm 37(6)$ & $14 \pm 13(13)$ & 0,88 \\
\hline Reestenose angiográfica, n (\%) & $1(7)$ & $1(14)$ & $0(0)$ & 0,2 \\
\hline
\end{tabular}

TABELA 2

Achados da tomografia de coerência óptica 28 dias após implante de stents Inspiron $^{\mathrm{TM}}$ e BioMatrix ${ }^{\mathrm{TM}}$ em artérias coronárias porcinas

\begin{tabular}{lcccc}
\hline & $\begin{array}{c}\text { Total } \\
(\mathbf{1 4} \text { stents })\end{array}$ & $\begin{array}{c}\text { Inspiron } \\
(\mathbf{7} \text { stents })\end{array}$ & $\begin{array}{c}\text { BioMatrix }^{\text {TM }} \\
(\mathbf{7} \text { stents })\end{array}$ & $\begin{array}{c}\text { Valor } \\
\text { de } \mathbf{P}\end{array}$ \\
\hline Área luminal, $\mathrm{mm}^{2}$ & $4,25 \pm 1,31(4,02)$ & $3,71 \pm 1,08(3,93)$ & $4,79 \pm 1,36(4,10)$ & 0,12 \\
Área do stent, $\mathrm{mm}^{2}$ & $5,73 \pm 1,01(5,62)$ & $5,31 \pm 0,66(5,50)$ & $6,15 \pm 1,16(5,91)$ & 0,12 \\
Área neointimal intrastent, $\mathrm{mm}^{2}$ & $1,48 \pm 0,61(1,54)$ & $1,61 \pm 0,57(1,71)$ & $1,36 \pm 0,66(0,95)$ & 0,47 \\
Área neointimal & $27 \pm 12(28)$ & $31 \pm 14(31)$ & $23 \pm 10(18)$ & 0,21 \\
Porcentual, \% & 2.184 & 1.283 & 901 & - \\
Hastes visibilizadas, $\mathrm{n}$ & $6(0,27)$ & $4(0,31)$ & $2(0,22)$ & 0,69 \\
Hastes em malaposição, $\mathrm{n}(\%)$ & $0(0)$ & $0(0)$ & $0(0)$ & $>0,99$ \\
Presença de trombo, $\mathrm{n}(\%)$ & & & & \\
\hline
\end{tabular}

tálicas e no desenho das malhas. O stent Inspiron ${ }^{\mathrm{TM}}$ é baseado no stent Cronus ${ }^{\mathrm{TM} 16,17}$ (Scitech Produtos Médicos Ltda.), um stent constituído de liga de cobalto-cromo L605, com espessura das hastes de $75 \mu \mathrm{m}$ e espessura da camada de polímero de $5 \mu \mathrm{m}$. O stent BioMatrix ${ }^{\mathrm{TM}}$ é baseado no S-Stent ${ }^{\mathrm{TM}}$, constituído de aço inoxidável 316L, com espessura das hastes de $112 \mu \mathrm{m}$ e espessura da camada de polímero de $10 \mu \mathrm{m}$.

Stents recobertos com polímeros bioabsorvíveis e com liberação de drogas antiproliferativas apenas do lado abluminal têm o potencial de acelerar a reendotelização e propiciar adequada adaptação ao vaso implantado. ${ }^{18}$
O stent Inspiron ${ }^{\mathrm{TM}}$, em desenvolvimento no Brasil, atualmente encontra-se em etapa de uso clínico inicial (fase II). Está em planejamento a realização de estudos clínicos randomizados (fase III) com a comparação de seu desempenho com outros tipos de stents farmacológicos já liberados para uso clínico no País.

Até o momento da redação deste trabalho os achados histopatológicos relativos aos stents implantados neste estudo em artérias coronárias porcinas ainda se encontravam em processamento. Esses achados à microscopia óptica irão detalhar as alterações que se seguiram ao implante desses stents e detectar eventuais diferenças desses dois tipos de stent. 


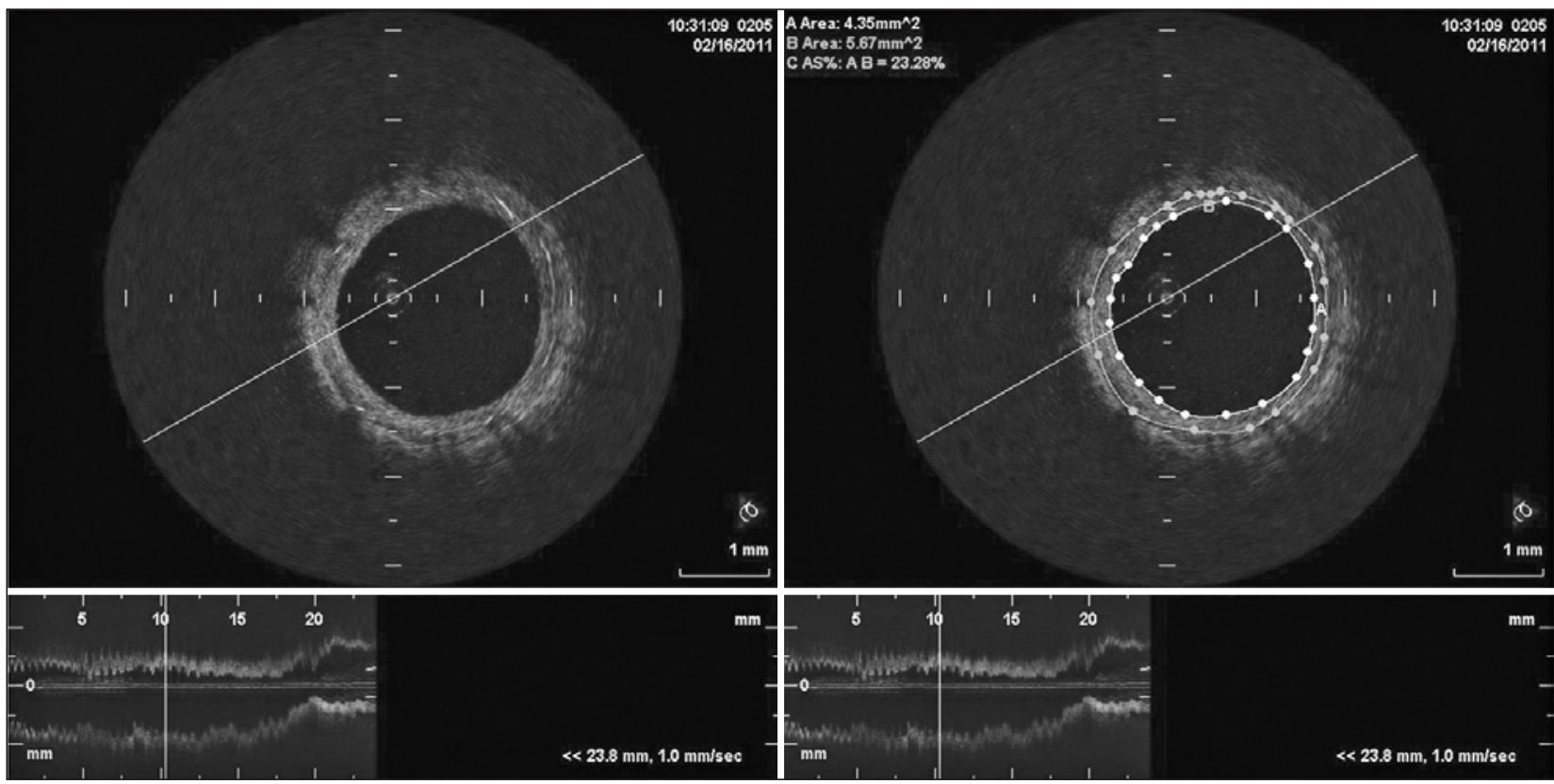

Figura 1 - No painel à esquerda, imagens representativas da tomografia de coerência óptica com corte transversal e reconstituição longitudinal de stent Inspiron ${ }^{\mathrm{TM}}$; no painel à direita, quantificação da área do lúmen $\left(4,35 \mathrm{~mm}^{2}\right)$, da área do stent $\left(5,67 \mathrm{~mm}^{2}\right)$ e da área porcentual de neoíntima $(23,28 \%)$.

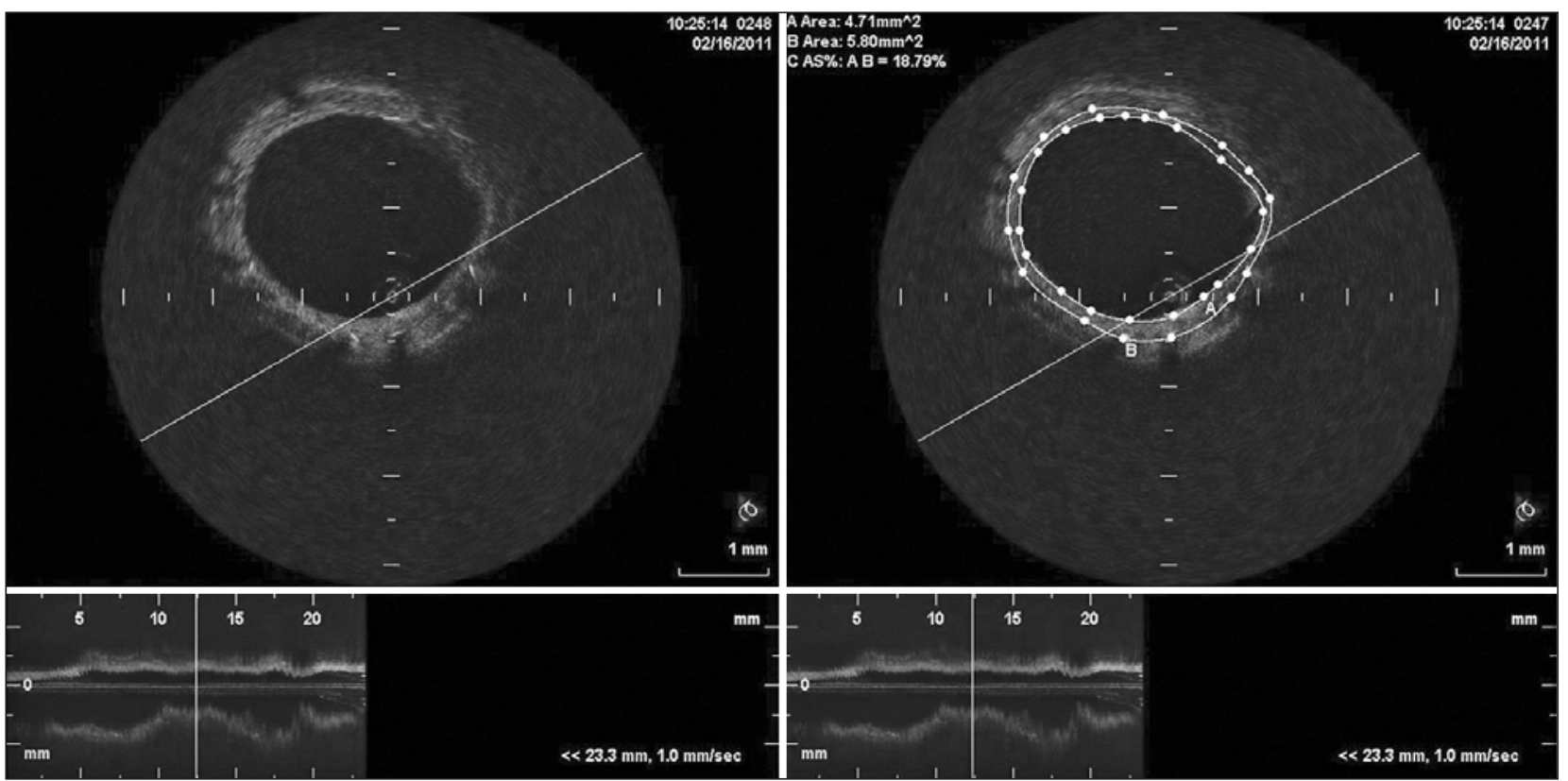

Figura 2 - No painel à esquerda, imagens representativas da tomografia de coerência óptica com corte transversal e reconstituição longitudinal de stent BioMatrix ${ }^{\mathrm{TM}}$; no painel à direita, quantificação da área do lúmen $\left(4,71 \mathrm{~mm}^{2}\right)$, da área do stent $\left(5,80 \mathrm{~mm}{ }^{2}\right)$ e da área porcentual de neoíntima $(18,79 \%)$.

\section{Limitações do estudo}

Este estudo apresenta as seguintes limitações: 1. O número amostral do presente estudo (análise total de 14 stents, 7 stents em cada grupo) não permite descartar a ocorrência de erro do tipo II ou beta. Um tamanho de amostra muito maior de animais seria necessário para efetivamente avaliar a superioridade de um stent em relação ao outro, que não foi o propósito deste estudo. 2. Na análise das imagens de tomografia de coerência óptica não foram mensuradas as espessuras neointimais de cada haste individualmente. Apenas três imagens transversais de cada stent foram analisadas para mensuração das áreas neointimais. 3. No presente 
estudo foram implantados stents farmacológicos em artérias coronárias de porcos não-ateroscleróticos. Estudos pré-clínicos com implante desses stents em artérias de animais com aterosclerose experimental (minipigs, coelhos) ou de animais diabéticos (porcos, ratos) podem responder com maior acurácia questões relativas principalmente à eficácia desses stents. ${ }^{19}$

\section{CONCLUSÕES}

Neste modelo experimental, os stents Inspiron ${ }^{\mathrm{TM}} \mathrm{e}$ BioMatrix $^{\mathrm{TM}}$, ambos recobertos em sua face abluminal com polímero biodegradável e com liberação de sirolimus e biolimus A9, respectivamente, demonstraram ser stents seguros, e à tomografia de coerência óptica os resultados evidenciaram semelhança no que se refere à área neointimal após 28 dias de implante em artérias coronárias porcinas.

\section{AGRADECIMENTOS}

Ao Prof. Fernando José Benesi, da Clínica de Bovinos do Centro de Pesquisa e Diagnóstico de Enfermidades de Ruminantes da Faculdade de Medicina Veterinária e Zootecnia da Universidade de São Paulo, pelos cuidados veterinários dispensados aos animais.

À sra. Leonora Loppnow, ao sr. Richard Barbosa da Silva e ao sr. Pedro Noiri Bisquilhari, técnicos do Serviço de Apoio à Pesquisa e Experimentação Animal, pelo auxílio técnico nos procedimentos experimentais.

Às agências de fomento FINEP, CnPQ e FAPESP, pelo apoio material e financeiro para execução desta pesquisa.

\section{SUPORTE FINANCEIRO}

O presente estudo faz parte do Programa de Desenvolvimento Nacional de Endopróteses Vasculares (stents) - PDNS, iniciado em 2004-2005. Conta com o suporte da Secretaria de Ciência, Tecnologia e Insumos Estratégicos (SCTIE)/Departamento de Ciência e Tecnologia (DECIT) do Ministério da Saúde (MS), do Conselho Nacional de Desenvolvimento Científico e Tecnológico $(\mathrm{CNPq})$ e da Financiadora de Estudos e Projetos (FINEP) do Ministério da Ciência e Tecnologia (MCT). O desenvolvimento do processo de corte a laser de stents conta com o apoio da Fundação de Amparo à Pesquisa do Estado de São Paulo (FAPESP; Forma de Apoio: Inovação Tecnológica - Pesquisa Inovativa na Pequena e Micro Empresa - PIPE).

\section{CONFLITO DE INTERESSES}

Celso K. Takimura e Francisco R. M. Laurindo são consultores científicos da Scitech Prod. Médicos. Pedro A. Lemos é consultor científico da Scitech Prod. Médicos, faz palestras eventuais, nacionais e internacionais, pelas empresas Boston Scientific e Cordis, e é integrante do Boston Scientific Latin America SCIMAB (Scientific Medical Advisory Board). Os demais autores de-
Avaliação pela TCO dos Stents Inspiron ${ }^{\mathrm{TM}}$ vs. BioMatrix ${ }^{\mathrm{TM}}$

claram não haver conflito de interesses relacionado a este manuscrito.

\section{REFERÊNCIAS}

1. Morice MC, Serruys PW, Sousa JE, Fajadet J, Hayashi EB, Perin $M$, et al. A randomized comparison of a sirolimuseluting stent with a standard stent for coronary revascularization. N Engl J Med. 2002;346(23):1773-80.

2. Moses JW, Leon MB, Popma JJ, Fitzgerald PJ, Holmes DR, $\mathrm{O}^{\prime}$ Shaughnessy $\mathrm{C}$, et al. Sirolimus-eluting stents versus standard stents in patients with stenosis in a native coronary artery. $\mathrm{N}$ Engl J Med. 2003;349(14):1315-23.

3. Stone GW, Ellis SG, Cox DA, Hermiller J, O'Shaughnessy C, Mann JT, et al. One-year clinical results with slow-release, polymer-based, paclitaxel-eluting TAXUS stent: The TAXUS IV trial. Circulation. 2004;109(16):1942-7.

4. Stone GW, Midei M, Newman W, Sanz M, Hermiller JB, Williams J, et al.; SPIRIT III Investigators. Comparison of an everolimus-eluting stent and a paclitaxel-eluting stent in patients with coronary artery disease: a randomized trial. JAMA. 2008; 299(16):1903-13.

5. Leon MB, Mauri L, Popma JJ, Cutlip DE, Nikolsky E, O'Shaughnessy $C$, et al. A randomized comparison of the ENDEAVOR zotarolimus-eluting stent versus the TAXUS paclitaxel-eluting stent in de novo native coronary lesions. J Am Coll Cardiol. 2010;55(6):543-54.

6. Stone GW, Teirstein PS, Meredith IT, Farah B, Dubois CL, Feldman RL, et al. A prospective, randomized evaluation of a novel everolimus-eluting coronary stent: the PLATINUM trial. J Am Coll Cardiol. 2011;57(16):1700-8.

7. Byrne RA, Mehilli J, lijima R, Schulz S, Pache J, Seyfarth M, et al. A polymer-free dual drug-eluting stent in patients with coronary artery disease: a randomized trial vs. polymer-based drug-eluting stents. Eur Heart J. 2009;30(8):923-31.

8. Chamié D, Costa Jr JR, Abizaid A, Silva JFA, Feres F, Mattos LM, et al. Stents liberadores de sirolimus com e sem cobertura polimérica: análise seriada com angiografia e ultrassom intracoronariano tridimensional. Rev Bras Cardiol Invasiva. 2009; 17(1):20-30.

9. Serruys PW, Ormiston JA, Onuma Y, Regar E, Gonzalo N, Garcia-Garcia HM, et al. A bioabsorbable everolimus-eluting coronary stent system (ABSORB): 2-year outcomes and results from multiple imaging methods. Lancet. 2009;373(9667): 897-910.

10. Missel E, Abizaid A, Cesar F, Centemero MP, Mattos LA, Feres $F$, et al. Análise volumétrica tardia após implante de stents eluidores de sirolimus versus biolimus A9. Rev Bras Cardiol Invasiva. 2008;16(1):37-43.

11. Windecker S, Serruys PW, Wandel S, Buszman P, Trznadel $\mathrm{S}$, Linke $\mathrm{A}$, et al. Biolimus-eluting stent with biodegradable polymer versus sirolimus-eluting stent with durable polymer for coronary revascularization (LEADERS): a randomized noninferiority trial. Lancet. 2008;372(9644):1163-73.

12. Lemos PA, Laurindo FRM, Morato SP, Takimura C, Campos CA, Gutierrez PS, et al. Stent coronário de liga cobaltocromo concebido no Brasil: achados histológicos preliminares em modelo experimental porcino. Rev Bras Cardiol Invasiva. 2007;15(4):378-85.

13. Campos CAHM, Takimura CK, Gregores GB, Sarmento CA, Fioretto ET, Laurindo FRM, et al. Redução neointimal com stent com polímero biodegradável e sirolimus desenvolvido no Brasil: resultados preliminares em suínos. In: Anais do $30^{\circ}$ Congresso da SOCESP; 2009; São Paulo, Brasil. São Paulo: Sociedade de Cardiologia do Estado de São Paulo; 2009. p. 25.

14. Takimura CK, Watanabe IS, Campos CAHM, Laurindo FRM, 
Ferreira CN, Gutierrez PS, et al. Avaliação por microscopia eletrônica de varredura da endotelização pós implante de stent farmacológico Scitech em artérias ilíacas de coelhos. Rev Bras Cardiol Invasiva. 2010;18 Supl 1:27.

15. Colégio Brasileiro de Experimentação Animal (COBEA). Princípios éticos na experimentação animal. São Paulo: COBEA; 1991.

16. Campos CAHM, Ribeiro EE, Lemos PA, Obregon A, Ribeiro $H$, Spadaro AG, et al. Resultados clínicos iniciais do primeiro stent de cromo-cobalto concebido no Brasil. Rev Bras Cardiol Invasiva. 2009;17(3):314-9.

17. Chamié D, Abizaid A. Stent Cronus: chegou o momento de adotarmos um stent nacional? [editorial]. Rev Bras Cardiol Invasiva. 2009;17(3):300-4.

18. Grube E, Schofer J, Hayptmann KE, Nickenig G, Curzen N, Allocco DJ, et al. A novel paclitaxel-eluting stent with an ultrathin abluminal biodegradable polymer. 9-month outcomes with the JACTAX HD stent. JACC Cardiovasc Interv. 2010; 3(4):431-8.

19. Nakazawa G, Nakano M, Otsuka F, Wilcox JN, Melder R, Pruitt $S$, et al. Evaluation of polymer-based comparator drugeluting stents using a rabbit model of iliac artery atherosclerosis. Circ Cardiovasc Interv. 2011;4(1):38-46. 